\title{
Assessing financial insecurity among common eye conditions: a 2016-2017 national health survey study
}

\author{
Grant L. Hom (D) ${ }^{1}$, Thomas B. Cwalina ${ }^{1}$, Tarun K. Jella ${ }^{1}$ and Rishi P. Singh ${ }^{2}$ \\ (c) The Author(s), under exclusive licence to The Royal College of Ophthalmologists 2021
}

\begin{abstract}
OBJECTIVE: To explore the prevalence and demographics of financial insecurity in individuals with eye disease in the United States. METHODS: This retrospective cross-sectional study analysed questions from the nationally representative 2016-2017 National Health Interview Survey (NHIS) with the eye conditions macular degeneration, diabetic retinopathy, glaucoma, and cataract. Data was analysed as a whole and then further analysed by condition. Evaluated topics indicated financial insecurity such as individuals reporting difficulty paying bills among eye conditions studied and by demographics.

RESULTS: Survey responses estimated that the overall prevalence of reporting problems paying or unable to pay bills were $12.49 \%$ (95\% C.I. 11.62-13.36\%) among patients with eye conditions. The overall prevalence of patients delaying care was $6.77 \%$ (95\% C.I. $6.17-7.36 \%$ ) and $17.06 \%$ (95\% C.I. 15.99-18.14\%) of individuals with eye conditions reported worrying about housing payments. Multivariable logistic regression revealed that demographics who more frequently had difficulty paying medical bills include individuals age 45-64 (3.33 aOR, C.I. 2.79-3.98, $p<0.001$ ), blacks (1.90 aOR, C.I., 1.48-2.45, $p<0.001)$, Hispanics (1.51 aOR, C.I. $1.07-2.12, p=0.020)$, and those $100-200 \%$ of the federal poverty line $(2.16$ aOR, C.I. $1.76-2.66, p<0.001)$ or below the poverty line (1.93 aOR, C.I. 1.48-2.53, $p<0.001)$.

CONCLUSION: There are several demographics with eye disease that self-report financial insecurity. There should be greater concern for financial insecurity among diabetic retinopathy and glaucoma patients. Ophthalmologists should consider engaging in proactive discussions with at-risk patients to reduce potential non-adherence secondary to financial insecurity.
\end{abstract}

Eye (2022) 36:2044-2051; https://doi.org/10.1038/s41433-021-01745-1

\section{INTRODUCTION}

Cost of care and income are among the many social factors impacting clinical treatment and outcomes [1, 2]. Medicare has spent $\$ 9719$ and $\$ 9934$ annually on each beneficiary receiving ranibizumab and aflibercept respectively [3]. In 2009, Medicare fee-for-service glaucoma costs were estimated to be around $\$ 228$ per person [4]. A cost analysis indicated yearly costs of glaucoma medications to range from $\$ 150.81$ for generic timolol maleate $0.5 \%$ (Falcon Pharmaceuticals, Ltd, Fort Worth, Texas, USA) to $\$ 873.98$ for a three-times-daily dose of Alphagan P $0.15 \%$ (Allergan, Inc, Irvine, California, USA) [5]. Lee et al determine that employer costs for diabetic retinopathy (DR) vary greatly among some subgroups with costs of care being higher in diabetic macular oedema employees (\$28606) versus non-diabetic macular oedema (\$16363), proliferative DR (\$30135) versus nonproliferative DR (\$13445), DR with photocoagulation (\$34539) versus without (\$16041), and DR with vitrectomy (\$63933) and without (\$17239) [6].

Previous ophthalmic literature in the United States and abroad indicates that income and cost of care may influence decisions to obtain care. Stagg et al determined that individuals in the United States with an income of over $\$ 100,000$ were $20 \%$ more likely to undergo cataract surgery at an ambulatory surgery centre than those with incomes less than $\$ 40,000$ annually (odds ratio 1.20;
$95 \% \mathrm{Cl}, 1.12-1.29)$ [7]. Furthermore, an Australian study establishes that more anti-vascular endothelial growth factor medicine was dispensed in neovascular age-related macular degeneration patients among bulk-billing clinics (17.0 mean injections) versus typical clinics (13.8 mean injections), indicating lower out of pocket expenditures are associated with higher adherence rates to therapy.

The Healthy People 2020 Vision objective establishes access to care as a key consideration for improving visual health of the United States [8]. A key aspect to patients appropriately accessing care is whether or not they feel they are in an adequate financial position to obtain care. Consequently, elucidating financial insecurity information among patients with common eye conditions may signal which patients may be basing healthcare decisions more so on cost rather than medical recommendation. With the National Health Interview Survey (NHIS), this study aims to explore the prevalence and demographics of financial insecurity in those with and without eye disease.

\section{METHODS}

This study was conducted across the 2016-2017 cycles of the National Health Interview Survey (NHIS), a cross-sectional nationally representative survey conducted annually by the National Center for Health Statistics

\footnotetext{
${ }^{1}$ Case Western Reserve University School of Medicine, Cleveland, $\mathrm{OH}$, USA. ${ }^{2}$ Cleveland Clinic Cole Eye Institute Center for Ophthalmic Bioinformatics, Cleveland, OH, USA 然mail: SINGHR@ccf.org
}

Received: 3 February 2021 Revised: 22 July 2021 Accepted: 3 August 2021

Published online: 23 August 2021 
(NCHS) (cdc.gov/nchs/). Using complex sampling design techniques such as stratification and clustering, the NHIS collects estimates of a wide range of health information for the civilian noninstitutionalized population of the United States. With over 600 unique questions included, the NHIS has been employed to analyse health concerns from a patient's self-reported perspective $[9,10]$. Topics previously studied within the ophthalmic space include disparities in eye care services, use of protective eyewear, and eyeglasses affordability [11-13]. Inclusion criteria for this study include survey respondents from individuals aged 18 or older. Responses pertaining to children were excluded with consideration to limited financial independence. This study was exempt from IRB approval due to the public nature of the data set. Informed consent was not required due to the retrospective nature and the IRB exempt status of this study. All study procedures adhere to the Declaration of Helsinki and all federal and state laws.

The NHIS includes questions in this study asking civilians about their financial security/well-being, with regards to both healthcare-related and non-healthcare-related expenditures. Such financial insecurity questions include the following topics: "problems paying or unable to pay medical bills, past 12 months"; "medical care delayed due to cost, past 12 months"; "worried about paying rent/mortgage/housing costs"; "worried about money for retirement"; "worried about paying monthly bills". The first two questions about "problems paying or unable to pay medical bills, past 12 months" and "medical care delayed due to cost, past 12 months" responses were structured in a yes/no format. The remaining three questions about financial worries served as a gauge of general financial stress and responses to these questions were structured in a four-step scale indicating the amount of worry: "not worried at all", "not too worried", "moderately worried", or "very worried" (Supplementary Appendix 1). The responses to the four-step scale questions about financial worries were then binarized into "not worried at all"/"not too worried" and "moderately worried"/"very worried" according to previous analysis of the NHIS to increase compatibility for statistical analysis [14].

These respondents from the NHIS were then filtered to only include those who reported having ever been told by a doctor or other health care professional that they had a common eye condition such as glaucoma, diabetic retinopathy, cataract, and macular degeneration. Within this subset of patients, only those who responded to the aforementioned financial security questions were included. The four conditions mentioned are the only eye conditions available for inclusion and information on other common eye conditions such as uveitis were not available in the NHIS database. This information is collected specifically by questions developed and utilized by NHIS, which is why data on ophthalmic conditions was only available in 2016-2017 as information gathering on ophthalmic conditions was not conducted in other years. Patients with the condition cataract included both patients with unoperated cataract and those with cataract surgery.

Analysis on these patients was first performed as a broad subset including all patients reporting to have the ophthalmic conditions cataract, diabetic retinopathy, macular degeneration, and glaucoma to understand trends of ophthalmic patients at large. Then, these populations were subdivided based on ocular condition to identify unique trends among each disease category.

\section{Statistical analysis}

$R$ version 4.0.2 (Boston, MA) was used for statistical analysis. All statistical calculations accounted for the complex sampling design of the NHIS using the R "survey" package (version 4.0). The overall proportions of each survey question response were calculated, including their $95 \%$ confidence intervals. The data was analysed for any crude differences in financial insecurity responses based on the independent variables included in the study with $95 \%$ confidence intervals being calculated for the breakdowns and statistical significance being determined with Pearson $X^{2}$ test with the Rao Scott adjustment [15]. In consideration of possible missing survey data, methodologies with imputed data have been used with the imputation being run five times [16]. To account for potential confounding factors, a multivariable analysis was conducted using logistic regression while controlling for all the independent variables to predict the financial insecurity responses. More specifically, one multivariable logistic regression model was run with age, race, sex, citizenship status, U.S. Birth status, geographic region, federal poverty level status, insurance status, marital status, level of highest educational attainment, and self-reported health status serving as independent variables and the dependent variable being a response to the financial insecurity question. From this regression, the adjusted odds ratios with $95 \%$ confidence intervals were calculated and statistical significance was determined using the Wald Test [17]. Statistical tests were two-sided, and significance was defined as $p<0.05$.

\section{RESULTS}

There were 175301 NHIS survey respondents in 2016-2017. A total of $12074,12094,11854,11839$, and 11857 patients within the ophthalmic cohort, which include conditions cataract, glaucoma, diabetic retinopathy, or macular degeneration, responded to NHIS survey questions relating to problems paying or unable to pay medical bills, past 12 months, medical care being delayed due to cost in past 12 months, worried about paying rent/mortgage/ housing costs, worried about money for retirement, and worried about paying monthly bills respectively. The demographics of responses are fairly consistent across survey questions. Based on the delayed cost survey question, (70.12\%, 95\% C.I. 68.97-71.27\%) of respondents were above the age of 65 and $76.25 \%(95 \%$ C.I. 74.60-77.89\%) were white and 9.28\% (95\% C.I. 8.31-10.25\%) were black (Fig. 1A). Females were more commonly respondents (58.66\%, 95\% C.I. 57.53-59.79) and majority of respondents are United States citizens (97.01\%, 95\% C.I. 96.50-97.53\%). A majority of participants were $200 \%$ or above the poverty line $(69.79 \%, 95 \%$ C.I. $68.41-71.18 \%)$, but a notable percentage were 100-200\% above poverty $(19.83 \%$, 95\% C.I. $18.86-20.79 \%)$ or $100 \%$ below

\section{A: Age and Race Demographics}
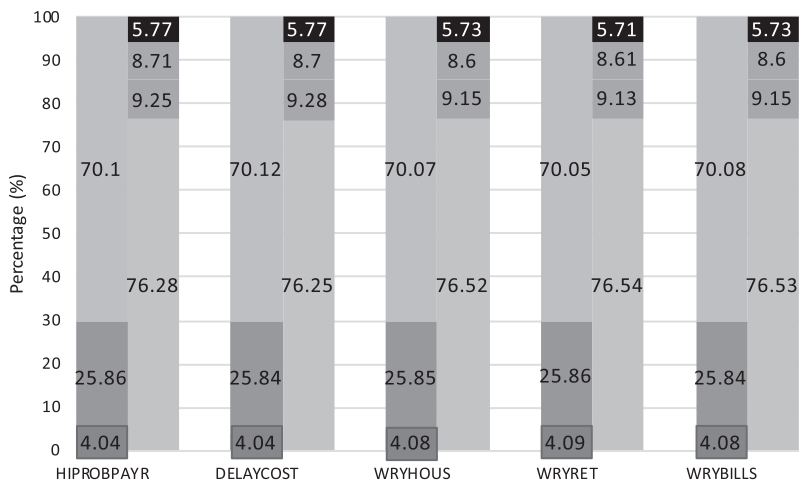

B: Poverty Level and Insurance Status

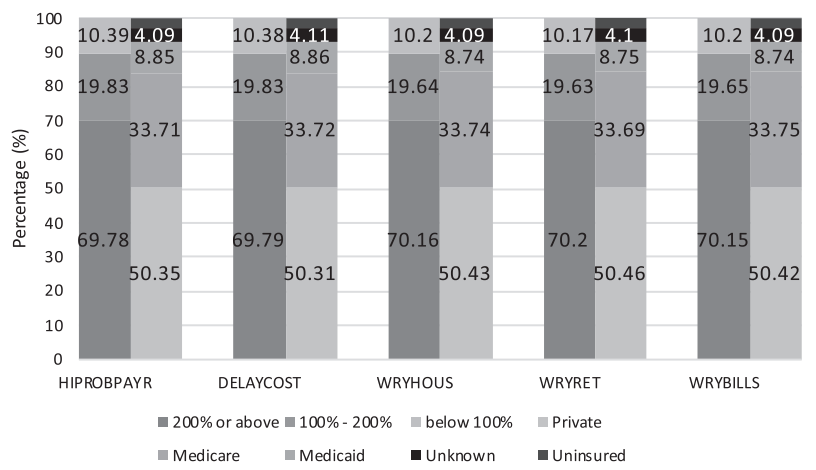

Fig. 1 Demographic Information of National Health Survey Respondents with Eye Disease. A Demographic information for age and race by percentage based on the National Health survey question. Figure 1B: Percentage of individuals by poverty level and Insurance status based on the National Health survey question. HIPROBPAYR "Problems paying or unable to pay medical bills, past 12 months"; DELAYCOST "Medical care delayed due to cost, past 12 months"; WRYHOUS. "Worried about paying rent/mortgage/ housing costs"; WRYRET "Worried about money for retirement"; WRYBILLS "Worried about paying monthly bills. 
poverty line (10.38\%, 95\% C.I. 9.52-11.24\%) (Fig. 1B). The majority of patients had private insurance (50.31, 95\% C.I. 48.72-51.89\%). More respondents had Medicare (33.72\%, 95\% C.I. 32.38-35.07) compared to Medicaid (8.86\%, 95\% C.I. 7.99-9.73\%).

Table 1 reports the percentage of patients who answered affirmatively to the ability to pay for medical bills in the past 12 months, medical care being delayed due to cost in the past 12 months, those worried about paying rent/mortgage/housing costs, worried about money for retirement, and worried about paying monthly bills respectively. For patients with an ophthalmic condition, the overall prevalence of reporting problems paying or unable to pay bills was $12.49 \%$ (95\% C.I. $11.62-13.36 \%)$ and the prevalence of delaying care was $6.77 \%$ (95\% C.I. $6.17-7.36 \%$ ). $17.06 \%$ (95\% C.I. 15.99-18.14\%) of individuals with eye conditions reported worrying about housing payments. 36.62\% (95\% C.I. $35.27-37.97 \%$ ) reported worrying over retirement and $23.75 \%$ (95\% C.I. $22.50-24.99 \%)$ reported worrying about bills. Notably, those over 65 are among the lowest to affirm difficulty in paying for medical bills in past 12 months $(8.09 \%$, C.I. $7.30-8.89 \%)$ or delaying care $(3.42 \%, 3.00-3.85 \%)$ compared to individuals in younger age brackets $(p<0.001)$. Furthermore, blacks and Hispanics had an increased rate of reporting difficulty to pay medical bills on time (23.98\%, C.I. $20.38-27.59 \%$ and $22.42 \%$, $18.02-26.82 \%$ for blacks and Hispanics respectively) or delaying medical care $(10.67 \%$, C.I. $8.25-13.09 \%$ and $11.58 \%$, C.I. $8.32-14.85 \%$ for blacks and Hispanics respectively) relative to their white counterparts $(9.87 \%, 9.01-10.73 \%$ and $5.48 \%$, 4.90-6.06\%) for problems paying and delaying medical care respectively $(p<0.001)$. Those below the federal poverty line frequently responded affirmatively to problems paying for medical bills $(23.48 \%$, C.I. $20.28-26.67 \%$ and delaying care (13.39\%, C.I. 10.94-15.84\%). Medicaid patients reported greater frequency of reporting difficulty paying medical bills $(18.61 \%$, C.I. $15.51-21.71 \%)$ and delaying care $(9.80 \%, 7.24-12.36 \%)$ compared to Medicare patients (11.66\%, C.I. $10.34-12.98$ and $5.82 \%$, C.I. 4.96-6.68 for difficulty paying bills and delaying costs questions respectively). The other factors that yielded statistically significant associations across all survey questions include U.S. Citizenship status, birth status, self-reported views on health status, and highest educational attainment.

Table 2 summarizes the adjusted odds ratios of affirmative answers to each survey question by demographic. Some notable demographics to experience elevated adjusted odds ratios (aOR) of having difficulty paying medical bills include individuals age 45-64 (3.33 aOR, C.I. 2.79-3.98, $p<0.001)$, blacks (1.90 aOR, C.I., 1.48-2.45, $p<0.001$ ) and Hispanics (1.51 aOR, C.I. 1.07-2.12, $p=$ $0.020)$, those $100-200 \%$ of the federal poverty line $(2.16 \mathrm{aOR}, \mathrm{C} . \mathrm{I}$. $1.76-2.66, p<0.001)$ or below the poverty line $(1.93$ aOR, C.I. $1.48-2.53, p<0.001)$. Uninsured patients had increase adjusted odds of being unable to pay for medical bills (1.87 aOR, C.I. 1.26-2.78) and delaying medical care due to cost (5.84 aOR, C.I. 4.00-8.52). Unique demographics that delayed medical care due to costs include females $1.24(1.02-1.52), p=0.035)$ and those who have single marital status (1.45 aOR, C.I., 1.45-1.81, $p<0.001)$.

\section{Financial insecurity among patients without ophthalmic conditions}

To understand if having ophthalmic conditions makes a noticeable difference in terms of ability to pay, a separate analysis was performed with patients without ophthalmic conditions. For the ability to pay question, $53.95 \%$ (C.I. 53.17-54.73\%) of individuals were between ages $18-44$ and $10.57 \%$ (C.I. 10.19-10.95\%) were $\geq 65$. The rate of affirmative response with difficulty paying bills was higher among the patients without eye conditions $(13.95 \%, \mathrm{C}$. I. $13.43-14.46 \%)$ than those with eye conditions $(p=0.003)$. There was a higher frequency of those delaying care among non-eye disease patients $(8.53 \%, 8.17-8.89 \%)$ relative to eye disease patients $(p<0.001)$.

\section{Financial insecurity among cataract patients}

Online Supplementary Tables $1 \mathrm{~A}$ and $1 \mathrm{~B}$ highlight the affirmative response rates and adjusted odds ratios to the survey questions of individuals reporting to have cataracts. 10678 individuals responded to the ability to pay question (Table 1A). The prevalence of inability to pay among cataract patients was $11.33 \%$ (C.I. 10.46-12.19\%) and delaying care was $6.05 \%$ (5.47-6.63\%). Notably, some of the following demographics who had cataract reported increase difficulty to paying medical bills: blacks (1.76 aOR, C.I. 1.31-2.35, $p<0.001$ ), individuals $100-200 \%$ (2.16 aOR, C.I. 1.73-2.70, $p<0.001)$ or below poverty line $(1.71 \mathrm{aOR}$, C.I. 1.25-2.34, $p=0.001$ ) (Table 1B).

\section{Financial insecurity among glaucoma patients}

Online Supplementary Tables 2A and 2B highlight the affirmative response rates and adjusted odds ratios to the survey questions of individuals reporting to have glaucoma. 1979 respond to the ability to pay question (Table 2A). The prevalence of inability to pay among glaucoma patients was $15.14 \%$ (C.I. 13.00-17.27\%) and delaying care was $8.76 \%$ (C.I. 7.14-10.38\%). Notably, some of the following demographics who had glaucoma reported increased difficulty to pay medical bills: Blacks (2.57 aOR, C.I. 1.63-4.07), $p<0.001)$ and Hispanics (1.97 aOR, C.I. 1.03-3.78, $p=0.042$ ), individuals $100-200 \%$ (2.40 aOR, C.I. $1.53-3.76, p<0.001)$ or below poverty line $(2.75 \mathrm{aOR}$, C.I. 1.61-4.69, $p<0.001$ ) (Table 2B).

\section{Financial insecurity among diabetic retinopathy patients}

Online Supplementary Tables $3 \mathrm{~A}$ and $3 \mathrm{~B}$ highlight the affirmative response rates and adjusted odds ratios to the survey questions of individuals reporting to have diabetic retinopathy. 606 individuals responded to the ability to pay question and a notable percentage of respondents were black (15.83\%, C.I. 11.89-19.76) and Hispanic (17.42\%, C.I. $12.93-21.91 \%)$. The prevalence of inability to pay among diabetic retinopathy patients was $25.47 \%$ (C.I. $21.43-29.51 \%$ ) (Table $3 \mathrm{~A}$ ) and delaying care was $14.56 \%$ (C.I. 10.68-18.43\%). Individuals who are no more than $200 \%$ above the poverty line (aOR 2.13, C.I. 1.07-4.27) or below the poverty federal poverty line (aOR 5.23 , C.I. 2.36-11.59) have a statistically significant increase in the likelihood of being unable to pay medical bills (Table 3B).

\section{Financial insecurity among macular degeneration patients} Online Supplementary Tables $3 \mathrm{~A}$ and $3 \mathrm{~B}$ highlight the affirmative response rates and adjusted odds ratios to the survey questions of individuals reporting to have macular degeneration. A total of 1466 responded to the ability to pay question and the majority of respondents were white (83.19\%). The prevalence of inability to pay among macular degeneration patients was $13.83 \%$ (C.I. 11.45-16.21\%) (Table 4A) and delaying care was 7.00\% (C.I. 5.42-8.58\%). Compared to the other conditions, it was notable that females had an increased adjusted odd of affirmatively responding that they had difficulties paying medical bills in the past 12 months (1.94 aOR, C.I. 1.24-3.04, $p$ $=0.004$ ) (Table 4B).

\section{DISCUSSION}

This study identifies the prevalence and at-risk groups of financial insecurity among patients with common ophthalmic conditions such as glaucoma, cataract, diabetic retinopathy, and macular degeneration. The overall prevalence of reporting problems paying or unable to pay bills among patients with eye conditions were $12.49 \%$. The overall prevalence of patients with an ophthalmic condition delaying care was $6.77 \%$. The overall prevalence frequencies are lower compared to patients without ophthalmic conditions who report problems paying bills (13.95\%) and delaying care $(8.53 \%)$. One explanation for these results is that the cohort without eye diseases tended to have a larger proportion of individuals in the age range 18-44 (53.95\%) compared to the cohort of patients with eye conditions (4.04\%). 
$\frac{\text { की }}{\frac{3}{3}}$

¿্ণ

¿্̀

రั

so

so

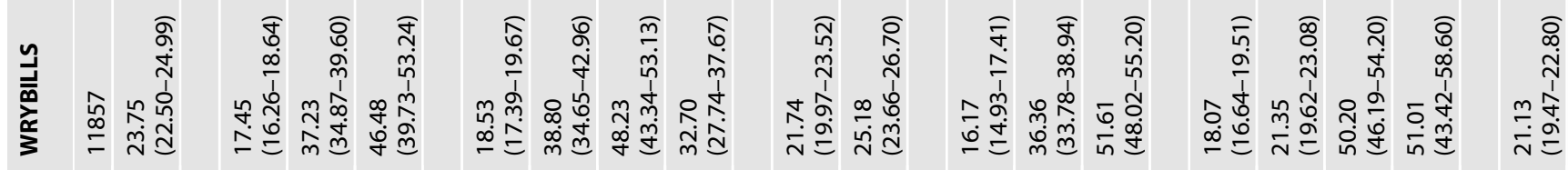
$\frac{\sqrt{2}}{\frac{1}{3}}$

¿্ণ

$\begin{array}{ll}\bar{c} & \bar{o} \\ \dot{v} & \dot{v}\end{array}$

¿্̀

¿্ৰ

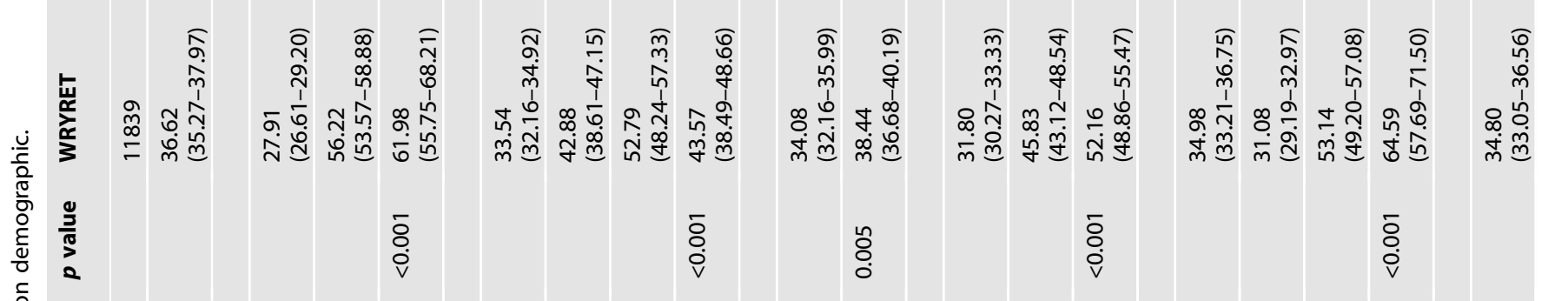

응

西

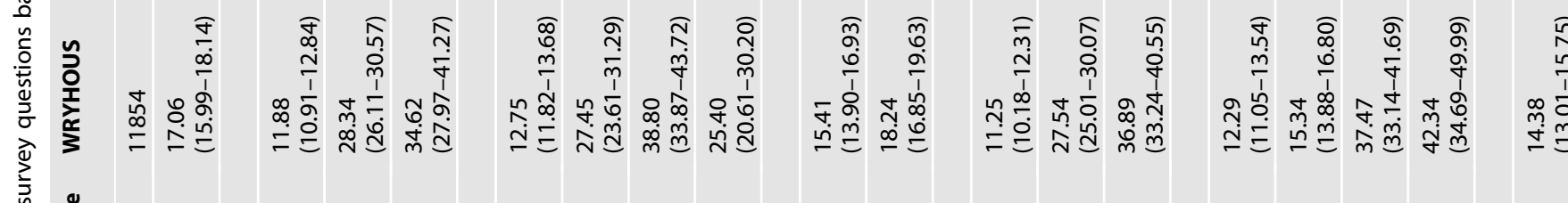

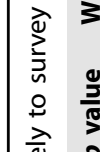

¿্̀

$\begin{array}{ll}\bar{o} & 0 \\ \dot{0} & 0 \\ 0\end{array}$

¿্̀

so

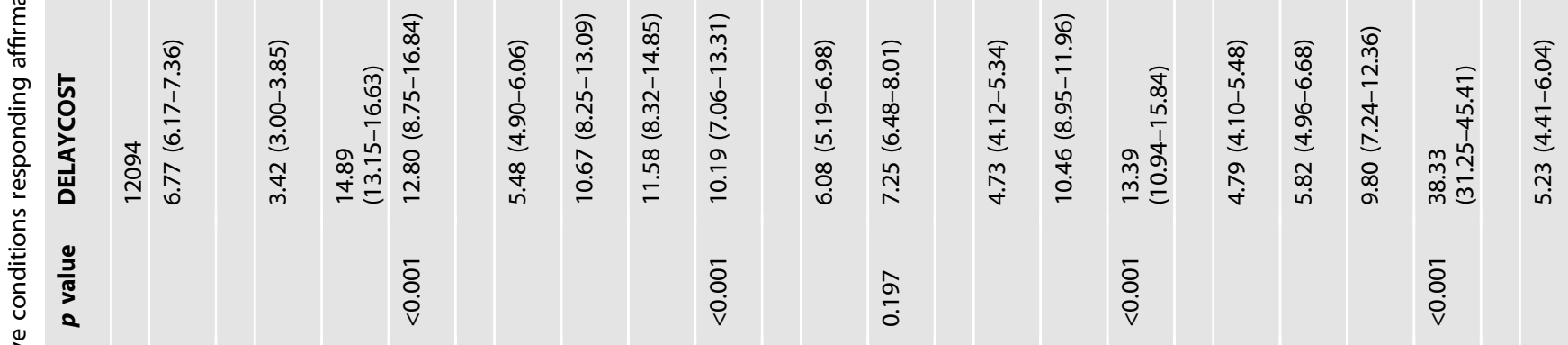

ชิ

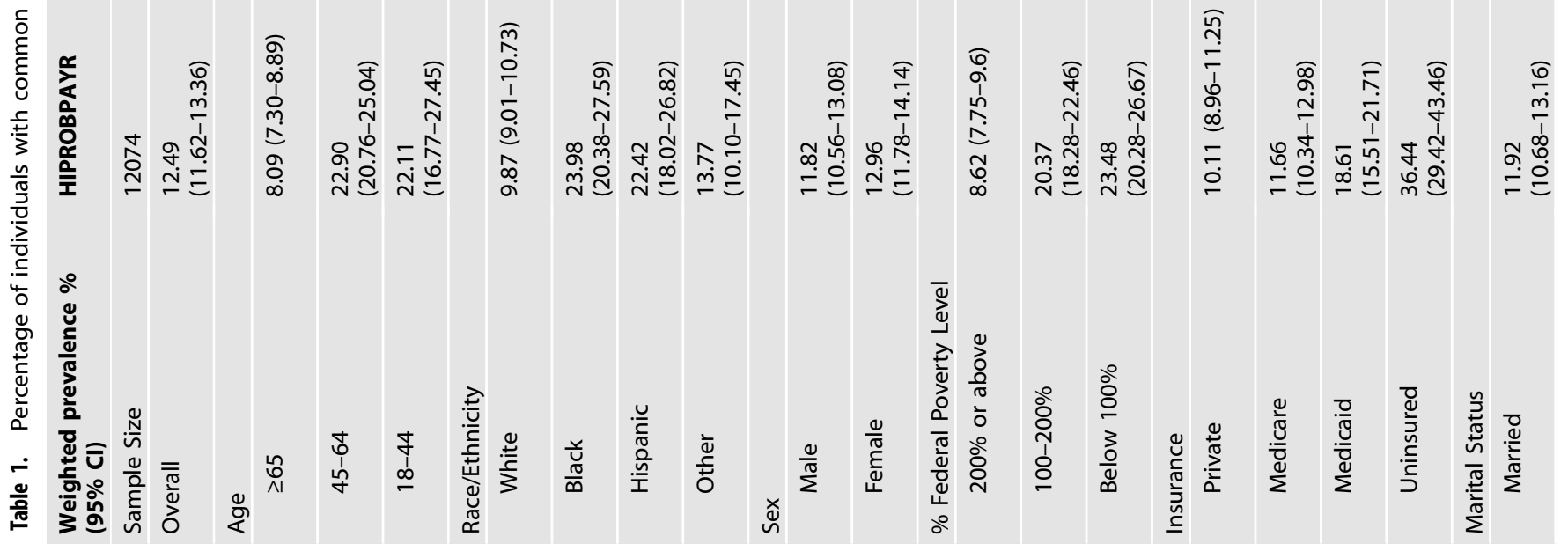


Consequently, the fewer years to earn income may contribute to higher frequencies of insecurity among patients without eye disease.

Stratifying the data based on disease yielded similar results with the prevalence of reporting problems to pay ranging from $11.3-25.47 \%$ and the prevalence of delaying care ranging from $6.37-14.56 \%$. Based on the entire cohort of patients including all four conditions, the study suggests that there are several factors that have distinguishable differences in reporting of financial stress and delay care such as race, age, poverty level, education status, citizenship status, and self-reported views on health. For example, Medicaid patients had a higher rate of affirmative answers to having problems paying medical bills and delaying care due to costs compared to Medicare patients. Controlling for all independent variables in this study, the results indicate that demographics at increased odds of being unable to pay for medical bills include being in the age bracket $45-64$, being black or Hispanic, being at the $200 \%$ level of the federal poverty line or below, being uninsured, and reporting lower quality health status. These findings generally are supported by previous literature on general socioeconomic data. The $>65$ age bracket is commonly supported by public health programs such as Medicare and consequently, less commonly experience financial difficulties relating to their healthcare due to decrease out-of-pocket expenditures [18]. The black and Hispanic demographics on average have been well reported to make a lower income and are supported by data indicating self-reported difficulty with housing payments and monthly bills [19]. Another notable finding is the increased aOR of females (1.24 aOR) affirmatively responding that they delay medical care due to costs. This coincides with knowledge that females on average make less than male counterparts [20]. Despite this knowledge, it has not been well established previously if these known variables translate to financial stress and have a possible influence on medical decision-making among ophthalmic patients.

The data on financial insecurity per ocular condition offers additional insights and permits for consideration of diseasedependent consideration such as different treatment burdens. Most notably, the diabetic retinopathy data reaffirms the burden of this condition on Hispanic and black individuals, but this data further highlights the stress that this type of a condition may have on finances as patients with this condition had the highest prevalence of reporting problems to pay medical bills $(25.47 \%)$ and the highest prevalence of delaying care (14.56\%). This high prevalence of financial insecurity may also signal difficulty affording healthy food options and gym memberships that can contribute to disease management and improve outcomes. Having single marital status has also been reported as a factor associated with delaying medical care due to cost among the cohort at large (1.45 aOR), but also the case of some specific disease cohorts such as the cataract cohort (1.34 aOR, Supplementary Table 1B). This makes sense as the ability to combine income may reduce financial stress.

While the study data presents self-reported financial stress among patients with conditions such as glaucoma, cataract, diabetic retinopathy, and macular degeneration, it is unclear if reported stressors over medical costs or delays in medical treatment directly relate to ophthalmic care because the NHIS survey does not allow us to elucidate who uniquely has a single ophthalmic condition and identify if that ophthalmic condition specifically is contributing to the financial stress. Notwithstanding, behaviours such as difficulty paying bills and delays in seeking medical care can adversely impact eye care. Ophthalmologists need to consider if affordability and financial concerns are potential contributors to suboptimal care such as loss to followup or refusal of treatment, particularly among demographics at increased risk of financial stress. 


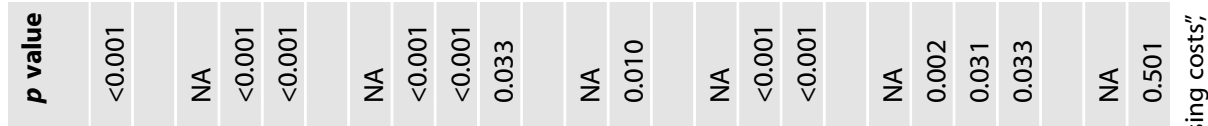

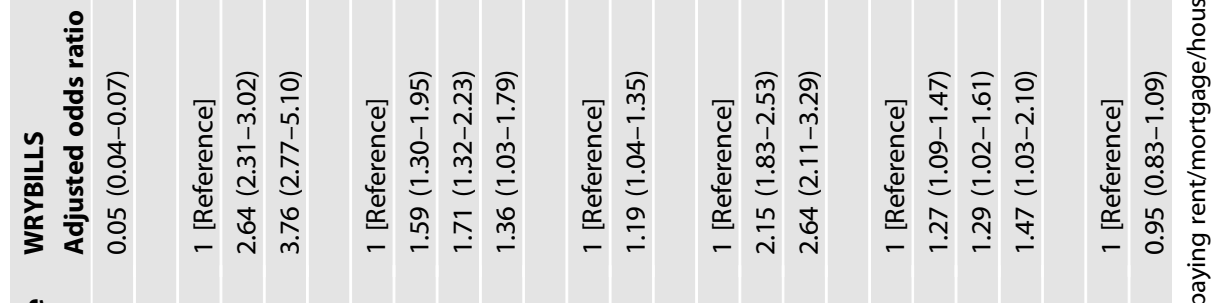

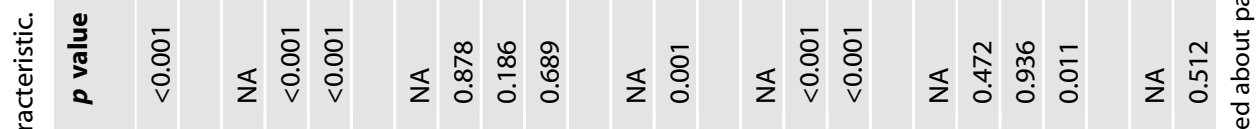

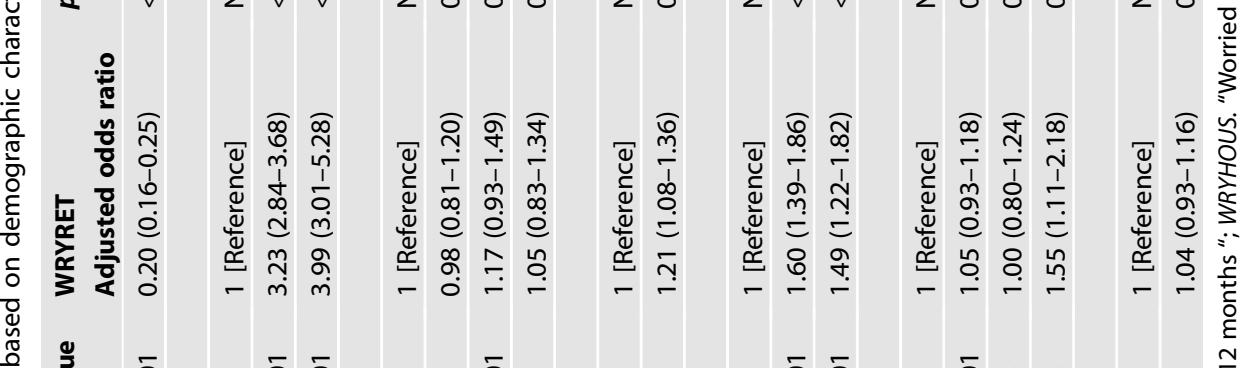

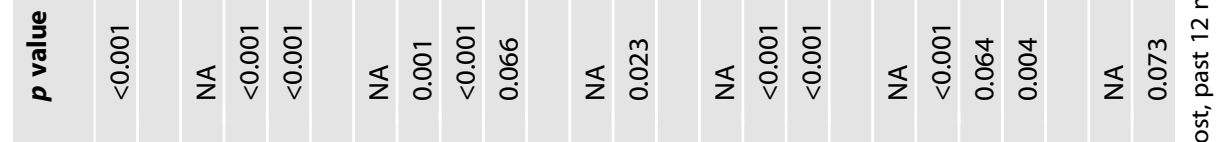

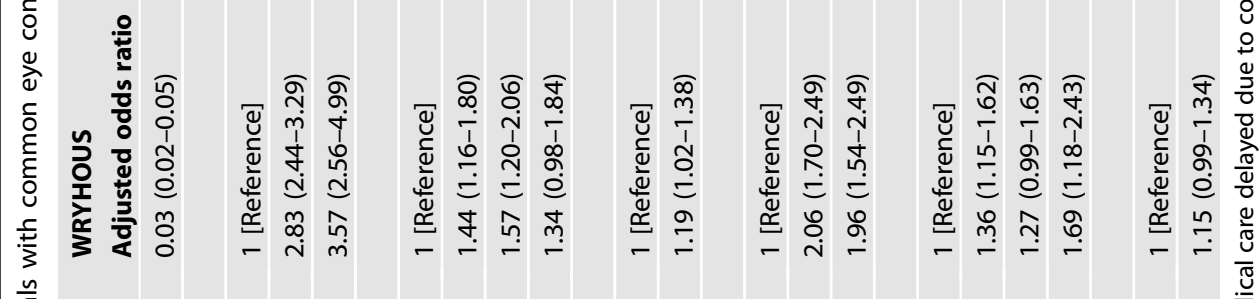

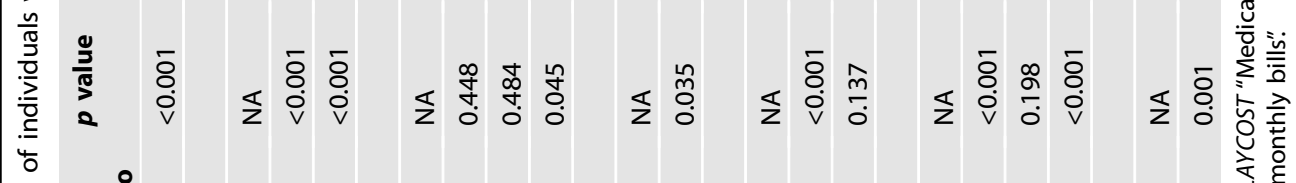

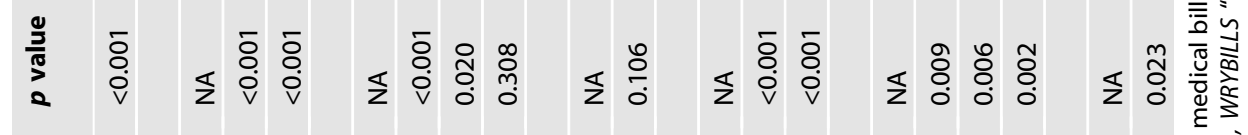

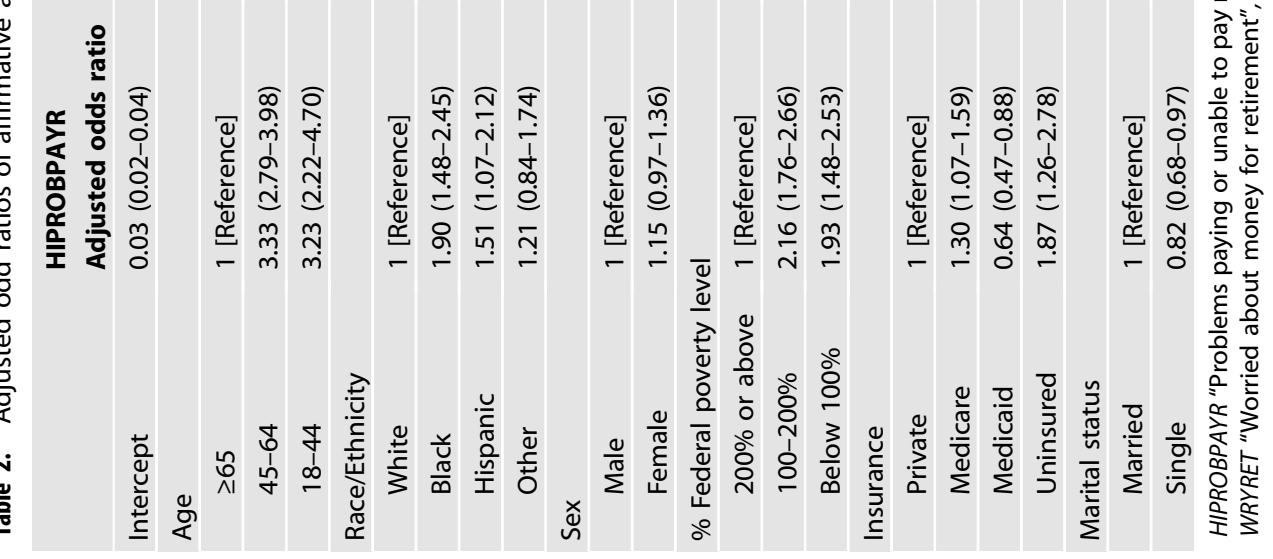


One unique consideration is that low reported overall health status had increased odds of having problems paying medical bills (aOR 2.57, $p<0.001$ ) and delaying medical care (aOR 3.02, $p<$ 0.001 ) in patients with ophthalmic conditions. Asking about overall health status during an ophthalmic appointment may be an indicator of individuals experiencing financial stress and can potentially be a tool for clinicians to gauge individuals who may be at risk for delaying medical care.

In healthcare broadly, several studies discuss improving and contextualizing care to those patients affected by lower socioeconomic factors and other social determinants. One option mentioned is to routinely screen for financial vulnerability across entire patient populations to identify and refer suitable candidates towards existing social services and safety net programs [21-23]. Others have commented, however, that universal screening may be costly, and offer minimal value wherever the availability or efficacy of subsequent interventions is limited [21, 22, 24]. Apart from screening, some institutions have begun validating and reporting healthcare-related financial stress as a patient outcome, increasing the possibility for standardized measurement and the subsequent evaluation of targeted interventions [25, 26]. Moreover, novel emerging technologies within ophthalmology such as virtual visits, artificial intelligence, and teleimaging may serve as possible mediums that can reduce financial stress related to ophthalmic appointments and reduce delays in medical care [27].

Some limitations should be noted in this study. Most notably, the NHIS survey does not offer insight into the severity of each ocular condition and relies on self-reporting for each condition. Conditions such as diabetic retinopathy and macular degeneration have varying treatment requirements depending on the severity of the disease, which may influence financial stress. The self-reporting of conditions can potentially lead to some under or over-reporting depending on each individual's understanding of their ocular history. For example, some patients may report having diabetic retinopathy if they went to see an eye doctor, but the patient could potentially have obtained routine monitoring without having the condition. The impact of multiple ocular comorbidities or other health conditions is unable to be further analysed. Moreover, the dataset is not fully complete as some questions had fewer responses than others, which was accounted for as best as possible via imputation.

A more recent consideration is the impact of the COVID-19 pandemic on finances and the impact of emerging economic changes has on financial stress. The study authors acknowledge that the pandemic possibly increases the likelihood of financial stress across a multitude of demographics, but still believe that this study's findings highlight broad trends that merit discussion among ophthalmologists and those advocating for improved patient centre care within ophthalmology.

Overall, this study adds to the socioeconomic literature within the ophthalmic space by examining a notable factor in the healthcare of patients: their finances and financial stressors. This study of a nationally representative cohort highlights that some demographics such as blacks, Hispanics, females, and individuals $200 \%$ or below the federal poverty line have an increased odds of experiencing financial stress. Additional demographics such as single individuals may also be subject to reporting financial stress relating to their healthcare. Consequently, while ophthalmologists should be willing to have discussions about the financial implications of their care with all patients, the demographics highlighted in this study may be subject to more frequent feelings of financial insecurity that could potentially impact their care, and conversations with these patients may be critical to appropriate management. Ophthalmologists and clinicians may need to play a proactive role in alleviating patient concerns to address potential non-adherence or loss to follow-up. Future studies should examine the direct impact of a given ocular condition on financial stress and can potentially examine additional financial considerations such as the impact of an ocular condition on professional opportunities or income. Furthermore, the relationship between the severity of these ocular conditions and financial stress should be examined more closely to better elucidate how possible treatment burden influences financial stress. Lastly, other aspects of care such as loss to follow-up and treatment differences can be examined in greater detail in the context of financial insecurity.

\section{Summary}

What was known before

- The prevalence of financial insecurity in ophthalmic patients has not yet been explored.

What this study adds

- This study explored the prevalence and demographics of financial insecurity in individuals with eye disease in the United States.

\section{REFERENCES}

1. Adler NE, Glymour MM, Fielding J. Addressing social determinants of health and health inequalities. JAMA - J Am Med Assoc. 2016;316:1641-2.

2. Artiga S, Hinton E. Beyond health care: the role of social determinants in promoting health and health equity. https://www.kff.org/racial-equity-and-healthpolicy/issue-brief/beyond-health-care-the-role-of-social-determinants-inpromoting-health-and-health-equity/.

3. Patel S. Medicare spending on anti-vascular endothelial growth factor medications. Ophthalmol Retin. 2018;2:785-91.

4. Quigley HA, Cassard SD, Gower EW, Ramulu PY, Jampel HD, Friedman DS. The cost of glaucoma care provided to medicare beneficiaries from 2002 to 2009. Ophthalmology. 2013;120:2249-57.

5. Rylander NR, Vold SD. Cost analysis of glaucoma medications. Am J Ophthalmol. 2008;145:106-13.

6. Lee L, Yu AP, Cahill KE, Oglesby AK, Tang J, Qiu Y, et al. Direct and indirect costs among employees with diabetic retinopathy in the United States. Curr Med Res Opin. 2008;24:1549-59.

7. Stagg BC, Talwar N, Mattox C, Lee PP, Stein JD. Trends in use of ambulatory surgery centers for cataract surgery in the United States, 2001-2014. JAMA Ophthalmol. 2018;136:53-60.

8. Vision | Healthy People 2020, https://www.healthypeople.gov/2020/topics-objectives/ topic/vision (accessed 3 December 2020)

9. Yabroff KR, Zhao J, Han X, Zheng Z. Prevalence and correlates of medical financial hardship in the USA. J Gen Intern Med. 2019;34:1494-502.

10. Mszar R, Grandhi GR, Valero-Elizondo J, Caraballo C, Khera R, Desai N, et al. Cumulative burden of financial hardship from medical bills across the spectrum of diabetes mellitus and atherosclerotic cardiovascular disease among nonelderly adults in the United States. J Am Heart Assoc. 2020;9:e015523.

11. Varadaraj V, Frick KD, Saaddine JB, Friedman DS, Swenor BK. Trends in eye care use and eyeglasses affordability: the US national health interview survey, 20082016. JAMA Ophthalmol. 2019;137:391-8.

12. Forrest KYZ, Cali JM, Cavill WJ. Use of protective eyewear in U.S. adults: results from the 2002 National Health Interview Survey. Ophthalmic Epidemiol. 2008;15:37-41

13. Zhang X, Beckles GL, Chou CF, Saaddine JB, Wilson MR, Lee PP, et al. Socioeconomic disparity in use of eye care services among US adults with age-related eye diseases: National health interview survey, 2002 and 2008. JAMA Ophthalmol. 2013;131:1198-206.

14. Butler SS, Winkfield KM, Ahn C, Song Z, Dee EC, Mahal BA, et al. Racial disparities in patient-reported measures of physician cultural competency among cancer survivors in the United States. JAMA Oncol. 2020;6:152-4.

15. Rao JNK, Scott AJ. On simple adjustments to chi-square tests with sample survey data. Ann Stat. 1987;15:385-97.

16. Simon AE, Uddin SFG. Trends in seeing an obstetrician-gynecologist compared with a general physician among U.S. women, 2000-2015. Obstet Gynecol. 2017;130:677-83.

17. Koch GG, Freeman DH, Freeman JL. Strategies in the multivariate analysis of data from complex surveys. Int Stat Rev/Rev Int Stat. 1975;43:59-78.

18. Barcellos $\mathrm{SH}$, Jacobson $\mathrm{M}$. The effects of medicare on medical expenditure risk and financial strain. Am Econ J Econ Policy. 2015;7:41-70. 
19. U.S. Census Bureau. Real Median Household Income by Race and Hispanic Origin: 1967 to 2017, https://www.census.gov/content/dam/Census/library/visualizations/ 2018/demo/p60-263/figure1.pdf (accessed 7 December 2020).

20. Bureau UC. Income, Poverty and Health Insurance Coverage in the United States, https://www.census.gov/newsroom/press-releases/2020/income-poverty.html (accessed 7 December 2020).

21. Garg A, Boynton-Jarrett R, Dworkin PH. Avoiding the unintended consequences of screening for social determinants of health. JAMA - J Am Med Assoc. 2016;316:813-4.

22. Davidson KW, McGinn T. Screening for social determinants of health: the known and unknown. JAMA - J Am Med Assoc. 2019;322:1037-8.

23. Fraze TK, Brewster AL, Lewis VA, Beidler LB, Murray GF, Colla $\mathrm{CH}$. Prevalence of screening for food insecurity, housing instability, utility needs, transportation needs, and interpersonal violence by US physician practices and hospitals. JAMA Netw open. 2019;2:e1911514.

24. Taylor LA, Tan AX, Coyle CE, Ndumele C, Rogan E, Canavan M, et al. Leveraging the social determinants of health: what works? PLoS One. 2016;11:e0160217.

25. de Souza JA, Yap BJ, Wroblewski K, Blinder V, Araújo FS, Hlubocky FJ, et al. Measuring financial toxicity as a clinically relevant patient-reported outcome: the validation of the COmprehensive Score for financial Toxicity (COST). Cancer. 2017;123:476-84.

26. Jagsi R, Ward KC, Abrahamse PH, Wallner LP, Kurian AW, Hamilton AS, et al. Unmet need for clinician engagement regarding financial toxicity after diagnosis of breast cancer. Cancer. 2018;124:3668-76.

27. Hom GL, Talcott KE, Muste JC, Singh RP. Remote imaging opportunities, innovations, and considerations for teleophthalmology. Am J Ophthalmic Clin Trials. 2021;4:1.

\section{AUTHOR CONTRIBUTIONS}

GLH was responsible for designing study protocol, data interpretation, and manuscript preparation. TBC was responsible for designing study protocol, data analysis, and manuscript review. TKJ was responsible for designing study protocol data acquisition, and manuscript review. RPS was responsible for designing study protocol and manuscript review.

\section{COMPETING INTERESTS}

RPS: Genentech/Roche (personal fees), Alcon/Novartis (personal fees), Apellis (grant), Graybug (grant), Zeiss (personal fees), Bausch + Lomb (personal fees), Ophthea (personal fees), Regeneron (personal fees). GLH, TBC, TKJ: None.

\section{ADDITIONAL INFORMATION}

Supplementary information The online version contains supplementary material available at https://doi.org/10.1038/s41433-021-01745-1.

Correspondence and requests for materials should be addressed to R.P.S.

Reprints and permission information is available at http://www.nature.com/ reprints

Publisher's note Springer Nature remains neutral with regard to jurisdictional claims in published maps and institutional affiliations. 